\title{
Le connessioni globali della colonia «scismatica» di Minorca (1743-1785)
}

\author{
Giampaolo Salice
}

Università di Cagliari

Les connexions globals de la colònia "cismàtica» de Menorca (I743-1785)

\section{Resum}

$\mathrm{Al}$ segle XVIII, molts estats europeus van mirar d'atreure hàbils colons estrangers cap als seus propis territoris, especialment aquells incorporats recentment. Els grecs van ser particularment escollits, gràcies a les seves notables capacitats comercials i marítimes i a les aptituds per fer de mediadors entre el Mediterrani occidental, el nord d'Europa i l'Imperi otomà. Aquest article se centra en la colònia grega establerta a la Menorca britànica i para una atenció especial en el paper desenvolupat per aquesta comunitat diaspòrica en la construcció de l'illa com un punt central en el procés de globalització.

Paraules clau: colons grecs, Menorca britànica, Maó, diàspora, globalització.

Las conexiones globales de la colonia «cismática» de Menorca (1743-1785)

\section{Resumen}

En el siglo XVIII, muchos estados europeos intentaron atraer a colonos extranjeros a sus territorios, en especial a los conquistados recientemente. Se apreciaba, en particular, a los griegos por sus habilidades comerciales y marítimas, así como por su capacidad para mediar entre el Mediterráneo occidental, el 
norte de Europa y el Imperio otomano. Este artículo analiza el papel que desempeñó la colonia griega establecida en la Menorca británica al hacer de la isla un punto central en el proceso de globalización.

Palabras clave: colonos griegos, Menorca británica, Mahón, diáspora, globalización.

\section{The global network of the "schismatic" colony in Menorca (I743-1785)}

\section{Abstract}

In the eighteenth century, many European States tried to attract skilled foreign colonists to their territories, especially those recently acquired. Greeks were especially wanted, thanks to their well-known commercial and maritime expertise, along with their ability to mediate between the western Mediterranean, northern Europe and the Ottoman Empire. This paper focuses on the Greek colony that settled in British Menorca, paying special attention to the role that this diasporic community played in rendering the Mediterranean island a central point in the globalization process.

Keywords: Greek colonists, British Menorca, Mahon, diaspora, globalization process.

\section{Introduzione}

Dopo avere condiviso per secoli i destini della Corona d'Aragona e della Spagna, nel i708 l'isola di Minorca passa sotto il controllo della Gran Bretagna. Il passaggio è internazionalmente sancito dal trattato di Utrecht (I7I3), che mette fine alla Guerra di Successione Spagnola. Gli storici ancora discutono sull'impatto che la dominazione britannica ha avuto sull' isola in un dibattito focalizzato sulle sue ricadute economiche: ${ }^{\mathrm{I}}$ se-

I. Martí Crespo, "Gibraltar i Menorca, les "illes britàniques" a la Mediterrània», in J. Albareda i Salvadó, A. Alcoberro i Pericay, eds., Actes del Congrés Els Tractats d'Utrecht. Clarors i foscors de la pau. La resistència dels catalans: 9-I2 abril 20I4, Museu d'Història de Catalunya; Departament de Cultura, Generalitat de Catalunya; 
condo alcuni studiosi il nuovo governo avrebbe innescato lo sviluppo agricolo, commerciale e infrastrutturale dell' isola, ${ }^{2}$ trasmettendo agli isolani un pragmatismo prima inesistente; 3 secondo altri, le riforme britanniche si sarebbero invece limitate ad accelerare processi di espansione commerciale e produttiva già attivi nell'isola ben prima della guerra di successione. ${ }^{4}$

Una delle riforme più significative adottate dai nuovi signori dell'isola è lo spostamento della capitale da Ciutadella a Mahón (I722). La città è dotata di uno dei più ampi porti del Mediterraneo occidentale e sotto i britannici diventa base militare, corsara e commerciale di primo rango. La presenza della flotta attiva una domanda di beni e servizi che l'economia locale non è in grado di soddisfare. C'è bisogno di forze esterne, che si cerca con successo di attrarre facendo di Mahón un porto franco. La liberalizzazione attrae ebrei, genovesi, greci e persino armeni. ${ }^{5}$

Questo saggio focalizza la sua attenzione sulla presenza greca a Mahón. Finora sono stati prodotti solo tre studi sul tema, due dei qua-

Institut Universitari d'Història Jaume Vicens i Vives; Universitat Pompeu Fabra, Barcelona, 20I5, p. 266.

2. A enfatizzare i benefici effetti della presenza britannica sono inizialmente gli stessi inglesi: John Armstrong, The History of the Island of Minorca, Davis \& Reymers, London, I756. Successivamente, Francisco Hernández SAnZ, La colonia griega establecida en Mahón durante el siglo XVIII, Imprenta de Manuel Sintes Rotger, Mahón, I925.

3. Román PIÑA Homs, Las instituciones de Menorca en el siglo XVIII: el fondo documental de Francesc Seguí, Caja de Baleares Sa Nostra, Palma de Mallorca, I986, pp. I5-I6.

4. Miquel Angel Casanovas Camps, "La Transformació d'una economia insular. El cas de Menorca: I600-I920", Butlleti de la Societat Catalana d'Estudis Històrics, I2 (200I), pp. I45-6o.

5. Così, ad esempio, nel 1748 marinai greci e armeni vengono ingaggiati dai britannici per il trasporto di libri attraverso il Mediterraneo. Cfr. The National Archives, Kew, England (NA), Admiralty, and Ministry of Defence, Navy Department: Correspondence and Papers (ADM), I06-I057. 
li risalenti alla prima metà del secolo scorso. ${ }^{6}$ Nessuno di questi ha inquadrato la colonia minorchina nel contesto della diaspora greca, fenomeno migratorio entrato nella riflessione storiografica internazionale solo negli ultimi decenni. Una riflessione che ha definito la diaspora come spazio policentrico di appartenenza, generato dalle relazioni tra le comunità, le colonie, gli individui che, oltre a sentirsi parte di un unico gruppo etnico-religioso e/o culturale, condividono la condizione della dispersione. ${ }^{7}$ Proprio le reti diasporiche, sviluppandosi fin dalla prima età moderna attraverso le frontiere istituzionali e culturali, hanno operato come strumento di mediazione tra autorità politiche e tra mercati diversi, tra sensibilità religiose e culturali anche se in conflitto, finendo col giocare un ruolo centrale nella formazione di network di relazioni su scala globale. ${ }^{8}$

6. Francisco Hernández Sanz, La colonia griega establecida en Mahón durante el siglo XVIII; Frederick Henry Marshall, "A Greek community in Minorca», The Slavonic and East European Review, II, n. 3I (1932), pp. I00-I07. Più recente è la tesi dottorale di Pedro Pablo Moreno Lucas Torres, «La colonia griega de Menorca a través de los documentos notariales de Mahón (1756-1802)», Universidad de Granada, 20II. Da segnalare anche il breve scritto di José Luis Terrón Ponce, Los Alexianos. Una familia griega en la Menorca del siglo XVIII, Luis Ripoll, Palma de Mallorca, 198I. Cenni alla colonia greco-minorchina anche in Desmond Gregory, Minorca, the illusory prize: a history of the British occupations of Minorca between 1708 and I802, Associated University Presse, London and Toronto, 1990; Miquel Angel Casasnovas CAMPS, Història econòmica de Menorca: la trasformació d'una economia insular (I3002000), Moll, Palma de Mallorca, 2006.

7. Sul problematico concetto di diaspora, mi permetto di rinviare al capitolo introduttivo in Giampaolo SAlice, Colonizzazione sabauda e diaspora greca, Sette Città, Viterbo, 20I5.

8. Olga Katsiardi-Hering, "The Greek Diaspora: Geography and Typology», in S. I. Asdrachas, ed., Greek Economic History: Isth-Igth Centuries, vol. I, P.I.O.P., Athens, 2007, pp. 237-47; Dēmētrēs Tziovas, Greek Diaspora and Migration Since I700: Society, Politics and Culture, Ashgate, London, 2009; Gelina HarLaftis, «The "Eastern Invasion": Greeks in Mediterranean Trade and Shipping in the Eighteenth and Early Nineteenth Centuries», in M. Fusaro, O. Mohamed-Salah, C. Heywood, eds., Trade and Cultural Exchange in the Early Modern Mediterranean: Braudel's Ma-

Pedralbes, 37 (20I7), I33-I62, ISSN: O2II-9587, DOI: IO.344/PEDRALBES2OI7.37.7 
La vocazione alla mediazione ha reso le diaspore spazi principalmente commerciali, in grado però di veicolare anche competenze in campo agricolo e militare e in ambito artistico e intellettuale. Un capitale umano ricco e articolato che può essere investito nelle politiche di potenza che gli Stati europei perseguono nell'età dei mercantilismi. La propensione statale a sfruttare il potenziale offerto dalla diaspora greca è già evidente nella prima età moderna. ${ }^{9}$ La tendenza si rafforza nel Settecento. In cambio di agevolazioni fiscali e di una relativa libertà di culto, i greci offrono ai Paesi che li accolgono le chiavi d'accesso ai mercati levantini e la possibilità di tessere relazioni commerciali con i tanti porti toccati dalla dispersione diasporica. I greci offrono inoltre canali di dialogo privilegiati con l'Impero ottomano e sopratutto con l'emergente Impero russo. ${ }^{10}$

ritime Legacy, I.B. Tauris, London, 20I0, pp. 223-52. Sul ruolo delle diaspore nella costruzione di reti globali di scambio cfr. Mark J. Miller, «Global Diasporas: An Introduction, and: The Politics of Migration (review)", Journal of World History Io, n. 2 (I999), pp. 44I-47; I. Baghdiantz McCabe, G. Harlaftis, I. Pepelasis Minoglou, eds., Diaspora Entrepreneurial Networks: Four Centuries of History, Berg, Oxford; New York, 2005; Richard L. Kagan, Philip D. Morgan, Atlantic Diasporas: Jews, Conversos, and Crypto-Jews in the Age of Mercantilism, I500-I800, JHU Press, Baltimore, 2009.

9. Si vedano ad esempio i profughi sefarditi impegnati nel Brasile olandese, in Jonathan Israel, "Jews and Crypto-Jews in the Atlantic World Systems, I500-I800", in R.L. Kagan, P.D. Morgan, eds., Atlantic Diasporas Jews, Conversos, and Crypto-Jews in the Age of Mercantilism, I500-I800, JHU Press, Baltimore, 2009, pp. 3-17; sui tentativi di insediamento dei moriscos in Italia cfr. Bruno Pomara Saverino, «La Diaspora dei Moriscos in Italia: storie di mediatori, schiavitù e battesimi», Storia Economica XVII, n. I (20I4), pp. I63-93 e, specialmente in Toscana, cfr. Cesare SANTus, «Moreschi in Toscana. Progetti e tentativi di insediamento tra Livorno e la Maremma (I6IOI6I4)", Quaderni Storici, n. 3/2013 (2013), pp. 745-78.

Io. L'universo umano che viene solitamente definito "greco» è caratterizzato da forte eterogeneità interna e da confini labili, sottoposti a mutamento costante. Sulla complessità del termine cfr. Mathieu GrenET, «La Fabrique communautaire. Les Grecs à Venise, Livourne et Marseille, v. I770-v. I830", tesi dottorale, European University Institute, Florence, 20I0, pp. 26-62. 
Sono principalmente queste le ragioni per le quali i greci trovano accoglienza nei principali scali portuali del mondo: da Marsiglia a Livorno, da Amsterdam a Calcutta, passando per Londra, Vienna, Budapest, a Odessa e per Venezia, Trieste, Napoli. La diaspora greca non approda solo nelle città di mare. Genova, Toscana, Prussia, Russia, Regno di Sardegna, tra Seicento e Settecento, hanno bisogno anche di agricoltori per trasformare i propri territori spopolati e impaludati in popolose aree di produzione agricola. Il "personale» diasporico non forma dunque un gruppo omogeneo. Anche per questo la dispersione genera insediamenti diversi, difficilmente riconducibili ad un unico ideal tipo.

La colonia di Minorca, che prende forma a metà del XviII secolo, incorpora caratteristiche comuni ad altre colonie diasporiche greche, ma con specificità che ne fanno per certi versi un caso unico. Fino alla conquista inglese, Minorca è un territorio integralmente cattolico. Il cattolicesimo minorchino, di matrice spagnola, considera l'omogeneità spirituale e la purezza razziale caratteristiche irrinunciabili. Le minoranze non cattoliche non sono dunque tollerate. La forza di simile ideologia è tale da resistere ancora nella Spagna borbonica, che pure nel Settecento si apre ai valori del Secolo e si impegna in una serie significativa di riforme d'ambito istituzionale, economico e demografico. Come le altre potenze europee, la Spagna borbonica desidera aumentare la popolazione nazionale, anche col concorso di coloni forestieri, ${ }^{\text {II }} \mathrm{i}$ quali però devono essere indubitabilmente cattolici .

Anche le élite minorchine di cultura cattolica e ispanica guardano con sospetto ai forestieri di altre religioni, che sempre più numerosi giungono a Mahón favoriti dalla protezione loro riservata dalla Corona britannica. I minorchini, agitati dal clero, provano ad opporsi. Gli strali dei cattolici colpiscono duramente i greci non appena questi manifestano l'intenzione di risiedere stabilmente nell'isola. Londra guarda

II. José Miguel Delgado BARRAdo, La génesis del proyecto repoblador de Sierra Morena, «Boletín del Instituto de Estudios Giennenses»/I78 (200I), pp. 303-329. 
con favore a simile richiesta, perché interessata a favorire il rafforzamento di un gruppo mercantile dinamico e ben collegato alle principali reti commerciali del Mediterraneo. Tuttavia - come vedremo - la colonia di Mahón nasce non solo grazie al governo britannico, ma anche alle pressioni dell'ambasciatore russo a Londra. Forte di queste connessioni «originarie», la colonia greca di Minorca si installa in un porto di grande rilevanza commerciale e militare, da dove tesse nuove reti di relazione di carattere commerciale e diplomatico estese all'intero Mediterraneo.

\section{Una fondazione policentrica}

Già all'indomani della firma del trattato di Utrecht, che all'articolo II riconosce alla Gran Bretagna il possesso di Minorca, si registrano i primi arrivi di forestieri sull'isola. Attratti dalle prospettive di profitto offerte dalla presenza di uno dei contingenti navali più imponenti del Mediterraneo, giungono al porto di Mahón mercanti genovesi, armeni, ebrei e greco-ortodossi. ${ }^{\mathrm{I2}}$ Tuttavia, solo nei primi anni Quaranta un pugno di famiglie greche chiede di risiedere stabilmente a Mahón. L'insediamento di una comunità in forma stabile implica però la costruzione di un luogo di culto.

Il 9 dicembre 1743 i mercanti greci inoltrano una supplica al vicario generale Miguel Barceló, vertice della gerarchia cattolica nell'isola. «Siendo Christianos Catholicos de la Iglesia griega — vi si legge — i greci necessitano di una chiesa dove «exercitar libremente su religion». ${ }^{13}$ Così, i ricorrenti godranno della stessa condizione dei connazionali

I2. Secondo una testimonianza tarda e non verificabile, tra i primi greci ad approdare sull'isola vi sarebbero alcuni esponenti della famiglia Alexiano, destinata a diventare punto di riferimento e guida dei mercanti greci di Minorca. Cfr. NA, Colonial Office (CO), I74/I, c. 98.

I3. Il documento è sottoscritto da Constantino Paleologo, Marcos Vatica, Nicola Malvasia y familia, Panago de George, Nicola Garato, Marco Tarassi y familia, 
stabiliti in Inghilterra, che «tienen publicamente capilla donde publicamente celebran los divinos officios y administran los sacramentos, sigun el ritu de la iglesia griega». ${ }^{14}$ Definendosi cattolici, i greci sembrano manifestare l'intenzione di organizzarsi secondo le procedure adottate dalle colonie greche installatesi in altri Paesi di culto latino, le quali hanno dato vita a comunità uniate che, pur conservando il rito orientale, sono formalmente sottomesse all'autorità del Papa. Il proposito sembra trovare conferma in un altro passaggio della supplica, in cui si sottolinea che l'esercizio del rito greco «es permitido, sigun differentes sacros consilios y por diferentes bullas de los summos pontifices y en particular por su santidad de Beneditto XIV, actual cabesa de la iglesia cristiana, como consta por la bulla que presentan». ${ }^{\text {Is }}$

Tuttavia, i mercanti ellenici devono essere consapevoli anche della particolare condizione nella quale si trova la chiesa cattolica minorchina, soggetta non all'autorità del pontefice romano, ma della Corona britannica. In questo quadro, i greci non avrebbero bisogno di ricorrere alla normativa pontificia, né di sottomettersi all'autorità del vicario generale dell' isola. Dunque perché fare appello al vicario? La procedura potrebbe essere stata suggerita dall'autorità britannica, impegnata a impedire ogni ingerenza romana sull'isola e a imporsi anche in campo spirituale, mettendo sotto tutela tutti i gruppi confessionali stanziati sul territorio, ma senza strappi, per consentire un'assimilazione graduale dei cattolici nel quadro ordinamentale britannico. Inoltre, la presenza a Minorca di minoranze non cattoliche, quindi autonome dal clero isolano, sembra ispirata, oltre che da ragioni commerciali, dal proposito di rafforzare i protestanti e di controbilanciare il peso delle élite lo-

Emanuel Paleologo, Nicola Danieto, Dimitri Filandri. Cfr. Arxiu històric de Maó (AHM), Universitat de Maó (UM), 390, s.n.

I4. AHM, UM, 390, s.n. Sulla comunità greco-ortodossa di Londra tra Sei e Settecento si veda Jonathan Harris, «Silent Minority: The Greek Community of Eighteenth-Century London», in D. Tziovas, ed., Greek Diaspora and Migration Since 1700: Society, Politics and Culture, Ashgate, London, 2009, pp. 3I-43.

I5. AHM, UM, 390, s.n. 
cali, che spesso si servono del cattolicesimo come strumento di mobilitazione generale, contro l'autorità britannica.

Dunque sono verosimilmente ragioni di opportunità a guidare l'agire dei greci. I mercanti sanno che il clero minorchino considera la purezza religiosa un elemento essenziale al godimento della piena libertà di culto tutelata dal trattato di Utrecht. E forse questa la ragione per la quale provano a interloquire col capo della chiesa isolana e, sempre nella supplica di cui si parla, lo informano di avere condotto a Minorca un sacerdote greco-cattolico. ${ }^{16}$ Marcos Vatica, uno dei ricorrenti greci, si è recato personalmente in Corsica a bordo dello sciabecco del mahonese Matheo Herpandes. Giunto ad Ajaccio, Vatica ha incontrato il sacerdote Giorgio Cassara, della colonia greco-maniota stanziatasi nell' isola nella seconda metà del Seicento. Nel I743 la colonia attraversa un momento di forte difficoltà. La loro cittadina Paomia è stata distrutta dai corsi insorti contro Genova, dopo che gli stessi maniotti si sono schierati a difesa della Repubblica. Circa settecento persone sono state così costrette a sfollare ad Ajaccio. Le condizioni di vita in città sono così precarie che i capi della colonia pensano di abbandonare l'isola. L'autorità genovese, venuta a conoscenza dei propositi maniotti, ha emesso un'ordinanza che vieta loro di lasciare la Corsica. La Superba non può permettersi di perdere un gruppo che sotto il profilo militare si è rivelato fondamentale nel quadro della ribellione corsa. ${ }^{17}$

Genova è informata anche delle mosse dei greci di Minorca. Nel novembre del 1743 il Senato avvisa tutti gli ufficiali di stanza in Corsica dell'imminente arrivo dell'ambasciata greco-minorchina di Vatica e ordina che le sia impedito di entrare in contatto coi greci di Paomia. La Superba è convinta che i minorchini vogliano provocare «la diserzione

I6. «... con la permission del exercio de la catholica religion griega» si legge nella supplica. Cfr. AHM, UM, 390, s.n.

17. I greci vengono organizzati dai genovesi in corpi militari. Si veda Emiliano Beri, Genova e il suo Regno. Ordinamenti militari, poteri locali e controllo del territorio in Corsica fra insurrezioni e guerre civili (I729-1768), Città del silenzio, Novi Ligure, 2OII, p. 9I-93. 
dei greci di Paomia o d'un loro prete per trasportare la colonia dei medesimi in Porto Maone». ${ }^{18}$ Tuttavia, quando l'informativa del Senato giunge a destinazione è già tardi. Giovanni Maria Manbitta, con dispaccio del 6 marzo I744 spedito da Calvi, scrive che fin da ottobre «si ancorò prima in questo Porto lo detto sciabecco e proseguendo poi il suo viaggio approdò nel golfo di Ajaccio, da dove, come ho saputo, fece disertare il prete Giorgio Cassara e si trasferì con detto sciabecco in Porto Maone ove attualmente si trova». ${ }^{19}$ A Minorca Cassara viene presentato a Barceló perché questi lo autorizzi a esercitare le funzioni di parroco nella erigenda chiesa greca. Barceló risponde il ıo dicembre 1743 negando il permesso, perché Giorgio Cassara — spiega il vicario - è fuoriuscito dal suo Paese senza il consenso del governo e del suo superiore diocesano. ${ }^{20}$

I mercanti greci di Minorca non demordono. Nel marzo del 1744 Marcos Vatica torna in Corsica e, sebbene non sia autorizzato a sbarcare ${ }^{21}$ chiede che siano consegnate a Paolo Maria Mariotti, vescovo di Sagona, ${ }^{22}$ le lettere con cui Giorgio Cassara chiede il permesso di amministrare i sacramenti a Minorca. ${ }^{23}$ Il magistrato di Corsica fa seguire da vicino i movimenti di Vatica. In assenza del vescovo - scrive il ministro genovese — «ho lasciate correre dette lettere e patenti alle

I8. Archivio di Stato di Genova (ASGE), Corsica, filza 935, s. n.

19. ASGE, Corsica, filza 935, s. n. La circostanza è confermata il 7 ottobre 1743 da un biglietto di Calicis, il quale informa che «in porto vi è uno sciabecco inglese procedente da Maone sopra il quale vi è un greco vestito all'inglese ... con parrucca nera rotonda e porta una canna d'India con pomo di cocco e dorme al bordo di detto sciabecco ... costui deve portarsi in Corsica per condurre via quei greci di Paomia o de' preti per fondare una colonia in detto Maone». Dello stesso tenore il dispaccio inviato da Gio Benedetto Speroni, commissario di Ajaccio, il 25 novembre 1743. ASGE, Corsica, filza 935, s. n.

20. AHM, UM, 390, s.n.

2I. "per la contumacia del bastimento procedente da luogo sospetto». ASGE, Corsica, filza 935, s.n.

22. Sagona è la diocesi nel cui territorio si trova la colonia greco-corsa di Paomia.

23. ASGE, Corsica, filza 935, s. n. 
mani del suo vicario Brunelli, al quale precedentemente ho fatto insinuare di ritenere dette lettere e patenti e negare le dimissorie col pretesto di non esserci il vescovo». ${ }^{24}$

A causa del diniego delle autorità genovesi e di quello del vicario generale di Minorca, Cassara non può né rientrare in Corsica, né stanziarsi a Minorca. A questo punto il sacerdote greco cerca una via d'uscita alternativa, aprendo una trattativa segreta col re di Sardegna Carlo Emanuele III, al quale propone un ambizioso progetto di ripopolamento con coloni greci della seconda isola del Mediterraneo. ${ }^{25}$ Le trattative approdano nel I746 a un contratto che prevede lo stanziamento di migliaia di coloni in arrivo dalla penisola del Mani, in Grecia. $^{26}$

Nel frattempo a Minorca la situazione sembra sbloccarsi. Il 28 novembre 1745, a Londra, il Privy Council accoglie la richiesta del principe russo Ivan Shcherbatov di concedere ai mercanti «de nacion griega» tutti i diritti già goduti dai nativi, insieme alla piena libertà di culto ortodosso. ${ }^{27}$ L'effetto della decisione è la naturalizzazione dei greci, che in quanto sudditi britannici potranno godere degli stessi diritti dei cattolici minorchini. Lo stato maggiore ecclesiastico di Minorca reagisce duramente. Si riunisce con una certa solennità il Is febbraio 1746 e vota unanime un documento che censura la decisione del governo britanni-

24. Ibidem.

25. SAlice, Colonizzazione sabauda e diaspora greca. I mercanti greci sono a conoscenza delle trattative tra i greco-corsi e il Regno di Sardegna, come emerge dalla già citata supplica al vicario Barceló. Cfr. AHM, UM, 390, s.n.

26. Il progetto non si concretizza perché i maniotti si rifiuteranno di fare professione di fede cattolica, prevista dall'accordo con Cassara e considerata essenziale dal sovrano sardo (e dai vescovi isolani). Nel 175I Cassara si impegna allora a trasferire l'intera colonia greco-corsa di Paomia in Sardegna. Cfr. SALICE, Colonizzazione sabauda e diaspora greca, pp. 45 e ss.

27. NA, CO, $174 / 2$, c. 26. Per l'iter burocratico che precede il pronunciamento del Privy Council cfr. Marshall, "A Greek community in Minorca», pp. IOO-IO2. 
co e minaccia dure ritorsioni. ${ }^{28}$ Se i greci erigeranno la propria chiesa «sin tener primero el permisso de su Santidad», i sacerdoti, dai pulpiti "de todas las parroquias de la isla», leveranno le loro voci per denunciare «que aquella iglesia no es christiana ny legitimamente elegida y que persona alguna no puede acudir a ella para recibir algun sacramento y ohir predicas...». ${ }^{29}$ Il clero vieta inoltre a qualsiasi prete cattolico di officiarvi senza la preventiva autorizzazione di Roma. ${ }^{30}$ Dunque, non solo viene ribadito che in materia spirituale i sacerdoti minorchini non riconoscono nessuna autorità se non quella del pontefice, ma sbarrano la strada anche a sacerdoti uniati come il greco-corso Giorgio Cassara.

Quest'ultimo sembra però avere ormai abbandonato l'idea di sistemarsi a Minorca, anche perché chi in un primo momento lo ha chiamato non ha più bisogno di lui. Non a caso, il 5 luglio I750, pochi mesi dopo l'avvio dei lavori di costruzione della chiesa greca di Mahón, il patriarca di Alessandria d'Egitto comunica al governatore di Minorca di avere autorizzato un curato (certo Daniel) a trasferirsi nella città portuale. ${ }^{31}$

L'aspra opposizione dei cattolici di Minorca non è dunque riuscita ad impedire l'innalzamento di una chiesa «scismatica» nell'isola. Una volta stabiliti, i greci di Mahón inviano dispacci ai loro «fratelli» disper-

28. AHM, UM, 390, s.n. c. 30 . Già il Io febbraio 1746 il vicario generale Miguel Morera aveva scritto al luogotenente Wynyard protestando perché la decisione assunta dal Privy Council costituiva una violazione del trattato di Utrecht. Cfr. NAL, CO, I74/2, c. 30 .

29. AHM, UM, 390, s.n.

30. Il clero minorchino si impegna a informare il Papa, così che la «cabeza que es de la universal iglesia catholica» possa adottare tutti i provvedimenti che riterrà opportuni. Il vicario invia la delibera di giunta anche alle locali autorità municipali. Così, l'8 marzo 1746 la Universitat di Mahón prende posizione contro la decisione del sovrano, considerata «tan impensada ... y sin tenir ningun havis» e definisce la prospettata apertura di una chiesa greca in città un fatto «tant perniciosa com pot considerare y aura vist per los capitols del dit Vicari general de quel tota la isla y particularment al pobla de Mahón estan mot cagiosos». Cfr. AHM, UM, 390, s.n.

3I. NA, CO, I42/2, cc. I2O e ss. 
si in diaspora per invitarli a trasferirsi a Minorca, dove agli ortodossi è consentito di professare liberamente e pubblicamente la propria fede e preservare i propri costumi..$^{32}$ Ad accogliere l'appello dei greco-minorchini è la potente famiglia degli Alexiano che — lo vedremo - giocherà un ruolo importante fino alla seconda metà del secolo. Ma le sirene greco-minorchine tornano ad echeggiare anche in Corsica. Nel I756 la Repubblica di Genova, non potendo più sostenere economicamente gli sfollati di Paomia, li autorizza a lasciare l'isola. Roma si preoccupa del destino di queste settecento anime, che rischiano di tornare allo «scisma» qualora dovessero trasferirsi in massa a Minorca o in altro luogo ortodosso. A impensierire Roma è anche un'eventuale migrazione verso un Paese cattolico, nel quale l'arrivo di una colonia così consistente potrebbe creare tensioni e pericolose convivenze coi cattolici di rito latino. Qualcosa di simili del resto sta avvenendo in Sardegna, dove Carlo Emanuele III di Savoia progetta di sistemare le famiglie greco-corse. In un primo momento, agli esuli viene assegnata l'isola di Sant'Antioco, nel Sud Ovest sardo. La decisione suscita reazioni molto accese da parte dei vescovi sardi, che trovano sostegno anche nel pontefice Benedetto XIV. ${ }^{33}$

Ma la Sardegna è solo una delle ipotetiche destinazioni. I greco-corsi vedono anche nella Toscana una possibile destinazione. L'arcivescovo di Pisa ne dà notizia al Papa con dispaccio del I4 giugno I756. I greci di Corsica — scrive il prelato — «cercarono di stabilirsi nella Maremma pisana per coltivare terreni. Il Granduca offrì loro le campagne dei vil-

32. Uno di questi dispacci arriva in Sardegna dove, dal I75I e per iniziativa di Giorgio Cassara, prende forma la colonia greca di Montresta, con coloni greco-corsi e maniotti. Cfr. SALICE, Colonizzazione sabauda e diaspora greca, pp. 93-96.

33. Stefano Pira, «Diaspore e tolleranza religiosa tra Sardegna e Mediterraneo: Benedetto XIV e la colonia dei Greci a Montresta», in S. Pira, ed., Nostos, Montresta e i Greci. Diaspore, emigrazioni e colonie nel Mediterraneo dal XVIII al XIX secolo, Diaspore 2, AM\&D, Cagliari, 2012, pp. 23-90. 
laggi di Sovana e Populonia, che però i greci rifiutarono». ${ }^{34}$ A quel punto, prosegue l'arcivescovo, «molti passarono in Minorca» dove il loro stanziamento fu reso impossibile «per non comunicare con quei loro nazionali scismatici, che prevalevano col dominio inglese». Per questa differenza spirituale, chiosa l'arcivescovo, la grande maggioranza di loro «si sono nuovamente rifugiati in Ajaccio, senza esigere dalla repubblica di Genova quelle condizioni vantaggiose che godevano in passato come colonia».35

\section{Un'esistenza precaria (I745-I756)}

Senza significativi innesti di coloni, quella di Minorca resta una colonia relativamente ristretta. Una condizione che non aiuta nel confronto con la schiacciante maggioranza dei cattolici, che a più riprese osteggia i forestieri ortodossi. Fin dal Io giugno I740 Andreu Costabella, procuratore fiscale dell'isola, denuncia al governatore britannico presunti illeciti commessi da parte dei mercanti greci. Secondo l'ufficiale, i levantini, che egli considera "poco timorosi di Dio e della giustizia temporale», avrebbero acquistato un'ingente partita di grano dal genovese Giovan Battista Magoni per poi tenerla in magazzino, al fine di rivenderla al momento giusto a prezzo di monopolio e con l'uso di pesi e misure alterati. ${ }^{36}$ L'episodio, di per sé marginale, è indicativo dell'irritazione e delle tensioni suscitate nella élite minorchina dal dinamismo economico della mercatura greca, che conquista uffici, prerogative, emolumenti prima riservati ai locali.

Denunce e resistenze non riescono però ad arrestare l'avanzata ortodossa. I greci, pur tra mille difficoltà, ottengono i terreni sui quali edificare la chiesa, il cimitero e le loro abitazioni. Il tempio, dedicato a

34. Archivio Storico "De Propaganda Fide" (ASPF), Scritture riferite nei congressi (SC), Italo-Greci, b. 4 (174I-1760), c. 35I.

35. ASPF, SC, Italo-Greci, b. 4 (174I-1760), c. 35I.

36. AHM, Cúria de la Reial Governació. Expedients Civils (RG), I65-2(7). 
San Nicola, diventa il cardine della comunità, sia in termini spirituali che urbanistici. Le case dei greci gli si dispongono intorno, mentre l'insediamento si sviluppa lungo Es Cós de Gracia, via di comunicazione che, come si legge in una targa ancora oggi presente all'inizio del percorso, è stata aperta nel I72I per velocizzare il collegamento tra il centro di Mahón e l'eremo di Gracia. ${ }^{37}$ Una strada che al suo termine meridionale incontra il Carrer de Gracia, l'asse viario nel quale da metà Settecento si insedia una colonia ebraica con la sua sinagoga. ${ }^{38}$ Greci ed ebrei danno così forma ad un nuovo distretto cittadino, corrispondente al margine esterno e più meridionale di Mahón.

I lavori di costruzione della chiesa greca, iniziati nel I749, si concludono dopo quattro anni; a finanziarli sono le donazioni delle famiglie più ricche della colonia. ${ }^{39}$ Tra queste spiccano certamente gli Alexiano, che si stabiliscono a Minorca alla fine degli anni Quaranta, ritagliandosi da subito un ruolo da protagonisti. Così, ad esempio, nel I753 il luogotenente Blackeney concede in affitto per trentun anni al capostipite della famiglia, Nicolas Alexiano, i terreni del capo di Mola, una salina in Fornells e un frutteto in Colarsega. ${ }^{\circ}$ Teodoro Alexiano, nipote maggiore di Nicolas, si è stabilito nell'isola dal I749. Al suo arrivo a Mahón, Teodoro è console dell'impero russo. L'incarico gli consente di distinguersi nella vita sociale ed economica della colonia, favorito anche dal sostegno dei due influenti zii, Nicolas Alexiano e Nicolas Malvasia.

In piccola scala, gli Alexiano adottano a Minorca il modus operandi tipico della diaspora greca del Settecento: si fanno interpreti degli inte-

37. AHM, UM, 382, c. 2.

38. Negli anni Ottanta del Settecento, in seguito alla riconquista spagnola di Minorca, vengono stilati diversi rapporti su numero e posizione degli immobili appartenuti ai greci e agli ebrei dell'isola. Questi documenti confermano che la gran parte dei greci risiedeva nei pressi della chiesa di San Nicola. Cfr. AHM, UM, 390, s. n.

39. L'elenco dei finanziatori e le relative quote donate è pubblicato in HeRnándEZ SANZ, La colonia griega establecida en Mahón durante el siglo XVIII, pp. I8-I9.

40. Terrón Ponce, Los Alexianos. Una familia griega en la Menorca del siglo XVIII, pp. 3-4. 
ressi commerciali e politici di diverse potenze statuali e provano a combinarli tra loro, traendo vantaggio dalle opportunità che simile dialogo può generare. Mentre Teodoro rappresenta la Russia come console, lo zio Nicolas gode di un rapporto privilegiato col governatorato britannico di Minorca. Presto Teodoro erediterà il ruolo rivestito nell'isola dagli zii, insieme ai rispettivi patrimoni, ${ }^{4 \mathrm{I}}$ ergendosi così a guida indiscussa della colonia e suo principale rappresentante nell'interlocuzione con i poteri pubblici, interni ed esteri, con i quali la colonia ha stabilito relazioni. ${ }^{42}$ In questo modo, il progetto di grandezza di una famiglia tende a sovrapporsi, fino a coincidere, con gli interessi della colonia che è chiamata a rappresentare. Il perseguimento di simili obiettivi impone la conservazione di un rapporto privilegiato con l'autorità britannica, della quale i greci diventano sostenitori decisi.

Nel 1756, allo scoppio della guerra dei Sette Anni, quando la flotta francese assedia Minorca, i coloni greci, organizzati da Teodoro Alexiano, si schierano a difesa delle insegne britanniche e combattono con grande determinazione. Dopo qualche mese i francesi hanno la meglio e occupano Minorca. Greci, ebrei e britannici vengono espulsi. I beni della colonia mercantile ortodossa di Minorca, inclusa la chiesa, vengono requisiti. Gli Alexiano sono tra i più colpiti dal tracollo britannico. Non resta che invocare il sostegno finanziario del sovrano in nome del quale hanno combattuto e perso tutto. Il 26 giugno 1759 il Privy Council esamina la richiesta di sussidio presentata da Giorgio Menie e Panagioty St. Maura, che durante l'assedio francese hanno servito come volontari

4I. In una supplica degli anni Ottanta, Nicolas Alexiano, fratello di Teodoro, illustra così le strategie patrimoniali della famiglia greca: «that as the manner of the greek nation is: the eldest branch generally possesses the entire property of the family and provides for the whole with grief and anguish of mind». Cfr. NA, CO, I74, I, c. 3 I2.

42. I due zii, Nicolas Alexiano e Nicolas Malvasia, aprono a Teodoro le porte del Consiglio dei greci, del quale fanno parte anche Nicola Paleologo e Zafyri Papodopolo. Cfr. Hernández Sanz, La colonia griega establecida en Mahón durante el siglo XVIII, pp. 22-23. 
nel forte di San Filippo. ${ }^{43}$ Il Privy Council accoglie la supplica e la inoltra al Tesoro. La stessa procedura è stata seguita dagli altri greco-minorchini privati dei loro beni; ${ }^{44}$ così anche Alessandro Alexiano, nativo di Grecia e fratello di Teodoro, il 2 febbraio 1762 lamenta la grande difficoltà economica nel quale versa a causa delle perdite subite. ${ }^{45}$

Al termine del conflitto, Minorca torna alla Gran Bretagna. Insieme ai britannici fanno rientro nell'isola i forestieri espulsi dai francesi. La famiglia Alexiano chiede la restituzione del patrimonio sequestrato dai nemici e un indennizzo per gli anni nei quali questo è stato destinato ad altri. ${ }^{46}$ Nicolas Alexiano jr., altro fratello di Teodoro, latore della richiesta, denuncia perdite per oltre diecimila sterline. ${ }^{47} \mathrm{Si}$ apre una vertenza della quale è rimasta traccia anche negli archivi minorchini. ${ }^{48}$ Proprio i documenti reperiti a Mahón ci informano che la causa ebbe luogo tra il 1763 e il I765. Gli Alexiano chiedono la restituzione sia delle case private dei greci sia della chiesa, che era stata assegnata dal comando francese alla truppa. Per dare forza alla petizione, Nicolas e

43. Alla petizione sono allegati i certificati firmati dal luogotenente Blackeney, che attestano la dedizione dei due greci durante l'assedio. Cfr. NA, Treasury (T), Treasury Board Papers and In-Letters (I)/393, s.n.

44. A fare domanda di sussidio/risarcimento sono, tra gli altri, George Stravangelo, Peter Michacliche, Nicolò Calimoriu, Demetrio Phacurely, Constantino Calamata, Stephon Solomon, Nicolo Rallo, Giacomo Cyprioto, Caramanij, George Bogeage, George Venerij, Panagioty St. Maura, Manuel Chretij, Athanasio Leffa, Estathio Mogademo, Alessandro Alexiano, Constantino Nicolo, Marcos Nicolo, George Lagarello. In NAL, Privy Council (PC) I/7/4. Un elenco dei greci che si sono battuti dal 19 aprile al 29 giugno 1763 con relative petizioni al Privy Council di Withewall anche in NAL, T, I/393.

45. NA, PC, $\mathrm{I} / 7 / 4$. Anche in questo caso è l'ex luogotenente Blakeney a certificare l'impegno di Alessandro Alexiano durante l'assedio francese. Su questa stessa petizione si veda anche NAL, T, I /364/56.

46. NA, CO, I42/I, c. 3 I2.

47. In questa occasione Nicolas Alexiano jr. dichiara che la sua famiglia abita a Maone dagli anni immediatamente successivi il trattato di Utrecht. Cfr. NAL, CO, I42/I, C. 3 I2.

48. AHM, Reial Gobernaciò (RG), I58 (I). 
Teodoro Alexiano rievocano i momenti nei quali, durante l'assedio, i due "convocaron todo el cuerpo [di greci] que residian en la isla y se offrecieron en tomar las armas ... en defensa de la Corona Britannica».49 Gli Alexiano evidenziano le gravi perdite seguite alla conquista francese: «fué obligado a salir con sus familias» dall'isola, lasciando case e patrimoni incustoditi "y han divagado por el mundo padeciendo las miserias y trabajos que la comprensión de Vuestra Excelencia puede contemplar». ${ }^{50}$

Volendo favorire il ritorno dei greci a Minorca, il governo fa loro restituire sia la chiesa sia le rispettive abitazioni. ${ }^{\text {sI }}$ Teodoro Alexiano torna a capo della restaurata colonia e riprende il progetto di grandezza personale e familiare, che culmina con la sua nomina a capitano del porto. A eleggerlo, nel I763, è James Johnston, appena giunto a Minorca in qualità di luogotenente governatore. ${ }^{52}$

La decisione di affidare a un greco il controllo del primo porto dell'isola esaspera l'avversione delle élite minorchine nei confronti dei forestieri. ${ }^{53}$ Le contestazioni sono tenaci e presto chiamano in causa lo stesso luogotenente che, poco fidandosi dei locali cattolici, fa affidamento principalmente sui forestieri. L'eco delle proteste è così forte da trovare spazio persino sulle pagine della stampa britannica di opposizione. Nel giugno del 1770 il periodico radicale The North Briton pubblica con

49. AHM, RG 158 (I).

50. Ibidem.

5I. Gli espropriati ottengono anche dei risarcimenti per gli anni di occupazione. Ad esempio, nel i774 la Universitat di Mahón paga un censo al greco Constantino Paliologo, come indennizzo per le case che gli sono state requisite durante l'occupazione francese. Le indennità corrisposte ai greci sono dunque a carico dei minorchini. Cfr. AHM, UM, I500.8I.

52. Per un quadro completo dei governatori e dei luogotenenti britannici a Minorca si veda Gregory, Minorca, the illusory prize: a history of the British occupations of Minorca between 1708 and 1802 .

53. Nel 1755 il luogotenente Blackeney, facendo seguito ai continui reclami dei locali, aveva ordinato a tutti i forestieri installati a Minorca di presentarsi e farsi registrare presso la cancelleria della Real Governazione. Cfr. AHM, RG, II6, c. I35. 
dovizia di particolari le petizioni e i ricorsi che le quattro universitats di Minorca hanno inviato al sovrano per mano di un sindaco appositamente nominato. ${ }^{54}$ Il giornale sfrutta la carica polemica dei documenti per attaccare il governo e accusarlo di amministrare con metodi tirannici le colonie, incitandole così a sollevarsi contro la Gran Bretagna..55

La scelta e l'operato di Teodoro Alexiano quale capitano del porto è uno dei principali motivi di contestazione. Le élite minorchine lo accusano di corruzione e abuso di potere. Alexiano avrebbe infatti preteso dai bastimenti il versamento di otto reali per l'ancoraggio, otto per diritti di ormeggio e quattro per lasciare il porto. Pedaggi considerati inaccettabili dalle autorità municipali, tanto più perché mai sono stati corrisposti in passato. Tra le righe delle denunce si percepisce come gli attacchi contro il capitano del porto siano ispirati da un più ampio disegno che punta a sbarazzarsi del protagonismo economico che i forestieri hanno riguadagnato col ripristino dell'autorità britannica. E non è certo un caso che l'accusa forse più pericolosa mossa contro Teodoro Alexiano riguardi lo smercio di passaporti e lasciapassare che il greco avrebbe messo in piedi a beneficio delle proprie tasche e dei tanti forestieri (specialmente genovesi, maltesi e greci) desiderosi di opera-

54. Nel xviII secolo Minorca conserva la gran parte delle istituzioni ereditate dal passato spagnolo. L'isola è divisa nei quattro distretti (terminos) di Ciutadella, Mahón, Alayor e Mercadal. Ciascun distretto è dotato di una bassa corte di giustizia, presieduta da un magistrato (bailo), e di un consiglio (Universitat). Ogni università distrettuale invia due deputati alla Università o Consiglio Generale, che ha sede a Ciutadella. La Università Generale rappresenta l'isola, interloquisce normalmente col governatore e, in caso di conflitto con questi, può rivolgersi direttamente al sovrano. Cfr. Antonio Ramis i Ramis, Noticias relativas á la isla de Menorca, vol. I, Imprenta de Serra, Mahón, I829.

55. Oltre che delle proteste minorchine, il periodico dà conto della dura presa di posizione dell'ambasciatore spagnolo a Londra per le misure adottate da Johnston a danno dei privilegi e delle consuetudini dell'isola ex spagnola. Cfr. John WiLkes, The North Briton from m. I to CCXVIII (June 5, I762-May II, I77I), voll. IOI-218, W. Bingley, London, I769, p. 43I. 
re sotto la protezione della flotta britannica. ${ }^{56}$ Una pratica che, secondo gli accusatori minorchini, colpisce al cuore il ceto mercantile autoctono, ma che viene ciononostante sostenuta dal luogotenente Johnston, il quale ha osato togliere la competenza su questo tipo di reati al tribunale del vice-ammiragliato per affidarla al fiscale Segui, un giurista minorchino filo-britannico. ${ }^{57}$

A Minorca gli accusati provano a difendersi. Una minuta non datata né firmata e conservata negli archivi della municipalità di Mahón riporta la testimonianza di un anonimo funzionario che dichiara di essere stato impiegato fin dal 1722 nel tribunale della Real Governazione..$^{58} \mathrm{Da}$ quando l'isola è diventata un dominio britannico — si legge nella minuta — la nomina del capitano del porto è sempre stata prerogativa dei governatori dell'isola, che l'hanno esercitata liberamente e senza l'intervento di nessun'altra autorità locale. La nota fornisce la lista dei nomi di coloro che hanno ricoperto il ruolo di capitano. Ultimo in ordine cronologico è proprio Teodoro Alexiano, che l'anonimo funzionario esalta per le notevoli competenze linguistiche. ${ }^{59} \mathrm{Si}$ tratta - si legge ancora nel documento — di una qualità fondamentale, dal momento che è «obligación peculiar del capitán del puerto» tenere il governatore costantemente aggiornato sugli arrivi dei bastimenti ed è per questo "precisamente necesario que la persona empleada en dicho oficio entienda los idiomas espańol, menorquin, francés e ingles, por ser cuasi todas las embarcaciones que entran en el puerto de una y otra de estas naciones». ${ }^{60}$

56. Teodoro Alexiano viene inoltre accusato di viaggiare continuamente tra Minorca e reggenze barbaresche senza patenti di sanità e senza il nulla osta delle autorità. Cfr. Robert Kirke, A Sketch of the Case and Sufferings of Mr. Robert Kirke, His Majesty's Late Consul at Algiers, s. Ed., London, I78I, pp. 88-I03.

57. Wilkes, The North Briton from m. I to CCXVIII (June 5, I762-May II, I77I), pp. IOI-2I8, p. 44I.

58. La minuta è evidentemente servita a preparare un originale da inviare a Londra. Cfr. AHM, UM, 390, s.n.

59. AHM, UM, 390, s.n.

6o. Ibidem. 
Sebbene nel 1765 il governo britannico ordini indagini sull'affaire dei passaporti, ${ }^{61}$ arrivando persino a richiamare Johnston a Londra per chiarimenti, il battage polemico innescato dai minorchini non sembra alla fine in grado di scalfire la posizione della famiglia Alexiano e della colonia greca. Nel 1769 , il posto di capitano del porto viene assunto da Nicolas Alexiano jr., ${ }^{62}$ destinato a diventare il nuovo capofamiglia alla morte del fratello di Teodoro (I775). Come in passato, gli Alexiano tornano così a imporsi come cognome guida della comunità greca di Minorca. Una posizione che si deve anche alla capacità della famiglia di muoversi dentro una rete di relazioni parentali, commerciali e politico-diplomatiche che, proprio in questi anni, assume dimensioni globali.

Proviamo a sintetizzare alcuni snodi salienti di questo protagonismo che si dispiega su scala planetaria. Già si è detto che quando nel I749 Teodoro Alexiano si stanzia a Mahón riveste l'incarico di console russo. A Minorca egli ottiene la cittadinanza britannica e costruisce un rapporto privilegiato col governatorato dell'isola. All'avvento dei francesi Teodoro lascia l'isola per l'Inghilterra dove viene ingaggiato come interprete da lord Albermarle, comandante in capo della spedizione che nel 1762 strappa alla Spagna La Havana e l'ovest di Cuba. ${ }^{63}$ Mentre partecipano su diversi scenari alla costruzione dell'impero britannico, gli Alexiano non rinunciano ai legami coll'impero russo, stretti già a metà Settecento. Sappiamo che nel 1775 Paniotto Alexiano, fratello di Teodoro, è comandante della nave da guerra russa San Paolo, ma è allo stesso tempo console britannico a Livorno, dove ha casa e dove nello

6r. Tra i testi sentiti c'è anche il fratello di Teodoro, Nicolas Alexiano. NA, CO, I74, I, cc. 98 e ss.

62. In un memoriale dei primi anni Ottanta, Nicolas dichiara che, in qualità di capitano del porto, ha percepito uno stipendio di cinque scellini al giorno. Cfr. NA, $\mathrm{CO}, \mathrm{I} 74, \mathrm{I}, \mathrm{c} .3 \mathrm{I} 2$.

63. Ioannis Hassiotis, Tendiendo puentes en el Mediterráneo: estudios sobre las relaciones hispano-griegas (ss. XV-XIX), vol. I, Centro de estudios bizantinos, neogriegos y chipriotas, Granada, 2008, p. 3II. 
stesso anno ospita il luogotenente di Minorca Johnston in visita. ${ }^{64} \mathrm{Da}$ Livorno Paniotto ha partecipato al quinto conflitto russo-turco insieme a numerosi altri greci assoldati dai russi. ${ }^{65}$

La collaborazione tra i greci Alexiano e i russi si mantiene solida nel corso del secolo e si rafforza dopo l'occupazione spagnola di Minorca del I9 agosto I78I. In occasione di questo secondo assedio, i greci si battono nuovamente al fianco dei britannici. A guidarli è Nicolas Alexiano, che il generale britannico Murray ha nominato comandante del corpo dei greci e dei turchi di stanza nel castello di San Filippo. Gli spagnoli hanno la meglio. Appena sbarcato nell'isola, il duca di Crillon, comandante delle truppe di invasione, ordina a greci ed ebrei di consegnare le armi $^{66}$ e ne fa confiscare le terre, le abitazioni e le altre proprietà. I beni requisiti vengono venduti e il ricavato distribuito alla truppa come ricompensa. ${ }^{67}$ Nicolas Alexiano viene condotto a Livorno come prigioniero di guerra insieme agli altri greci combattenti. Di là egli riesce ad approdare in Inghilterra dove si appella al sovrano in cerca di sostegno economico. ${ }^{68}$

Anche gli altri componenti della famiglia Alexiano (così come la maggior parte della colonia) tornano a disperdersi in diaspora: Alessandro, Paniotto e Antonio Alexiano, fratelli di Teodoro e Nicolas, si arruolano nell'esercito russo come ufficiali della marina imperiale; le sorelle Galitiza e Alessandra sposano rispettivamente il capitano della marina mercantile russa Nicolas di Giorgio e il capitano di marina Juan Ghiolma, nativo di Cefalonia e suddito veneziano. ${ }^{69}$

64. «Gazzetta Toscana», n. 4, I775.

65. Sulle gesta dell'Alexiano al comando della San Paolo cfr. «Notizie del Mondo", n. 46, 8 giugno 1773 .

66. AHM, Altres Arxius (AA), 5.I.8.I.

67. NA, CO, I74, I, c. 312.

68. NA, CO, I74, I, c. 3 I2.

69. Terrón Ponce, Los Alexianos. Una familia griega en la Menorca del siglo XVIII. 


\section{Diaspora e diplomazia}

Come abbiamo visto, le relazioni diplomatiche giocano un ruolo decisivo nella vicenda della colonia greca di Minorca, dalla sua fondazione fino alla scomparsa. Sono soprattutto le pressioni del principe Shcherbatov, ambasciatore russo a Londra, a consentire ai mercanti greci di stabilire la propria chiesa senza sottomettersi alle gerarchie cattoliche isolane. Negli stessi anni, proprio Shcherbatov, prima come ambasciatore in Spagna e poi in Gran Bretagna, ${ }^{70}$ si muove per attivare ogni canale utile a consentire ai russi l'ingresso nel Mediterraneo, lo stabilimento di saldi rapporti commerciali e diplomatici con le principali potenze dell'Europa occidentale e la costruzione di alleanze anti-turche.

L'impegno di Shcherbatov a favore dei greci di Minorca è dunque indicativo dell'importanza che l'isola occupa nella strategia complessiva della Russia. Una centralità che si apprezza specialmente nella partita diplomatica che la Russia gioca con la Gran Bretagna, che entrerà nel suo vivo nel $1763^{71}$ e nella quale Minorca viene continuamente chiamata in causa. ${ }^{72}$

Anche la Gran Bretagna è interessata a stabilire un rapporto positivo con la Russia, che potrebbe aiutarla a spezzare il suo isolamento europeo. In simile contesto, il favore concesso ai greci di Minorca può essere letto proprio come segnale dell'apertura britannica verso la Rus-

70. A. Alimento, K. Stapelbroek, eds., The Politics of Commercial Treaties in the Eighteenth Century: Balance of Power, Balance of Trade, Springer, New York, 2017, pp. I84 e ss.

7I. Matthew S. Anderson, "Great Britain and the Russo-Turkish War of I76874", The English Historical Review, 69, n. 270 (1954), pp. 39-58.

72. L'interesse russo per la base navale britannica emergerà anche quando Caterina II si dirà disponibile a garantire sostegno marittimo alla Gran Bretagna contro la Francia, la Spagna e le insorte colonie americane in cambio della cessione di Minorca. Cfr. Frank A. Golder, "Catherine II and The American Revolution», The American Historical Review, 2I, n. I (1915), pp. 92-96. 
sia che, proprio da metà secolo, si candida a suprema tutrice di tutti gli ortodossi.

Tuttavia, una formale alleanza tra i due Paesi non viene siglata, soprattutto perché i britannici non vogliono farsi trascinare in una guerra contro la Sublime Porta, che per Caterina II è invece il fronte decisivo nella corsa verso l'Europa Occidentale e il Mediterraneo. Una corsa che giunge ad una tappa decisiva con la vittoria della quinta guerra russo-turca (I768-I774) grazie alla quale la Zarina strappa la Crimea agli ottomani e la trasforma in un suo protettorato, spianandosi così la strada verso il Mar Nero e il Mediterraneo; garantisce a tutti gli ortodossi, anche se sudditi ottomani, la facoltà di navigare battendo bandiera russa; conquista il diritto di erigere una nuova chiesa ortodossa nel cuore di Costantinopoli: è la prima volta che succede dal I453 ed è una vera umiliazione per il sultano. ${ }^{73}$

Un simile successo politico-militare è il frutto del paziente lavoro diplomatico messo in campo dalla Russia negli anni precedenti il conflitto, al quale hanno contribuito in misura significativa anche i greci della diaspora che, presenti nelle principali città europee, hanno operato come veri e propri agenti russi all'estero, a sostegno degli interventi militari degli zar in Levante e delle sollevazioni filo-russe nella Grecia ottomana. ${ }^{74}$

La fondazione della chiesa e della colonia ortodossa di Minorca andrebbe dunque letta non solo come il frutto della politica commerciale e della tolleranza religiosa della Gran Bretagna, ma anche (forse sopratutto) come esito locale della strategia russa di tessitura di una rete globale di agenti diplomatico-commerciali. Minorca presenta poi caratteristiche specifiche che la rendono particolarmente attrattiva per i russi. In primo luogo è una base navale di grande rilevanza strategica;

73. Roderic H. Davison, «The "Dosografa” Church in the Treaty of Küçük Kaynarca", Bulletin of the School of Oriental and African Studies, University of London, 42, n. I (1979), pp. 46-52.

74. Franco Venturi, Settecento riformatore, vol. III. La prima crisi dell'Antico Regime (I768-1776), Einaudi, Torino, 1979, pp. 22-34. 
in secondo luogo è un dominio britannico. Due caratteristiche che fanno di Minorca uno spazio privilegiato di negoziazione tra russi e britannici, nel quale si discute e si progetta l'ingresso dei russi nel Mediterraneo, impossibile senza il sostegno della Gran Bretagna.

In una partita tanto delicata la colonia greco-minorchina svolge un essenziale ruolo di mediazione e operativo. Il più volte menzionato Teodoro Alexiano, console russo, suddito britannico, capitano del porto e capo della colonia ellenica di Mahón ${ }^{75}$ è tra coloro che si impegnano direttamente nel trasferimento degli squadroni navali russi dal Baltico al Mediterraneo, realizzato per la prima volta tra il 1769 e il $1773 .^{76}$

Favorendo la collaborazione tra russi e inglesi, i greco-minorchini guadagnano una posizione strategica nel quadro mercantile mediterraneo e globale, potendo operare sotto la duplice protezione di due potenze diplomatiche e militari di prima grandezza. È facile intuire quali vantaggi economici e politici possano derivare dalla conquista di una simile posizione.

Ma a spingere i greci di Minorca, che — ribadiamo - sono sudditi britannici, a lavorare anche per i russi è anche l'emergere di un primo sentimento filellenico, alimentato proprio dall'impero zarista. Negli stessi anni in cui la colonia di Minorca combatte per affermarsi, intellettuali come Evgenii Bulgaris, tra i più autorevoli esponenti dell'illuminismo greco e iniziatore del filellenismo, si installano nel cuore stesso dell'impero russo. Dopo essere fuoriuscito dalla Grecia, Bulgaris arriva a Pietroburgo e conquista la fiducia di Grigory Potemkin, negli anni in cui questi è amante e ministro di Caterina e progetta la colonizzazione interna dell'Ucraina e della Crimea. ${ }^{77}$ Insomma, greci e russi collaborano in tutti i settori nei quali Caterina promuove il rafforzamento dello Stato. In ambito marittimo e commerciale, i greci offrono

75. Anderson, "Great Britain and the Russo-Turkish War of I768-74", p. 45.

76. Il trasferimento avviene nel quadro della quinta guerra russo-turca.

77. Marc Raeff, Catherine the Great: A Profile, Springer, New York, 1972, p. 227. Sullo statista Potemkin si veda Simon Sebag Montefiore, Potemkin: Catherine The Great's Imperial Partner, Vintage Books, New York, 2005. 
perizia marinaresca; presenti in tutti i principali porti del Mediterraneo i greci sono in grado di fare da mediatori commerciali e culturali a tutela degli interessi russi. In campo agricolo sono migliaia i coloni greci assoldati dai russi per la ricolonizzazione della Crimea. ${ }^{78}$

Ma perchè i greci in diaspora possano operare a beneficio dei russi, debbono essere liberi da condizionamenti esterni. Nell'Europa cattolica, la professione di fede cattolica di fatto sottomette le colonie al controllo della Chiesa di Roma. A sua volta, la subordinazione al vescovo cattolico rafforza l'assoggettamento nei confronti del Paese ospitante.

Se l'ambasciatore russo a Londra si spende perché la chiesa greca di Minorca sia libera di professare il culto ortodosso, senza ingerenze da parte della gerarchia cattolica, è perché egli punta a mettere i coloni nelle condizioni di agire liberamente a favore della Russia. Per la stessa ragione, negli stessi anni, la Russia esercita pressioni anche sulla chiesa greco-uniate del porto franco di Livorno, per spingerla a sottrarsi al controllo cattolico e verso la fede ortodossa. La spregiudicata manovra russa allerta immediatamente l'alto clero toscano. Il I4 giugno I756 l'arcivescovo di Pisa Francesco Guidi informa il pontefice che «nella metà dello scorso mese ricevei lettera confidenziale del senatore Rucellai in cui mi accennava essere venuto da Vienna un foglio ... trovato contenere in sostanza di farsi divenire la chiesa Greca di Livorno Scismatica». ${ }^{79}$

L'alto prelato toscano, dopo avere allertato il Consiglio di Reggenza del Granducato, ${ }^{80}$ il 25 di maggio si è recato a Livorno per incontrare il

78. Matthew S. Anderson, "The Great Powers and the Russian Annexation of the Crimea, 1783-4", The Slavonic and East European Review, 37, n. 88 (1958), pp. I7-4I.

79. Archivio Storico di Propaganda Fide (ASPF), Scritture riferite nei congressi (SC), Italo-Greci, b. 4 (I74I-I760), c. 35I.

8o. Magistratura istituita nel 1737 da Francesco Stefano di Lorena, cui era affidato il governo del Granducato in sua assenza. Aveva competenze su tutti gli affari e agiva in base alle istruzioni del sovrano. Le riforme del 25 aprile 1739 affidavano al Consiglio di Reggenza la sorveglianza sull'amministrazione della giustizia, sulla conservazione dell'ordine pubblico, sul progresso dell'istruzione universitaria, delle arti e 
governatore Ginori. Dopo quasi due settimane di confronti — scrive il vescovo — «mi riuscì, grazie a Dio, d'illuminarlo, di schiarire li equivoci che prendeva, d'indurlo a sentire la parte dei greci cattolici, di stare cauto alla fallacie delli scismatici e di metterlo nelle giuste tracce di formare una vantaggiosa relazione alla reggenza cui indirizzata in questa stessa settimana sarà da lui stesso». ${ }^{81}$ Negli stessi giorni l'arcivescovo di Pisa incontra anche i greci cattolici della città per spronarli «a sostenere virilmente l'evidente ragione del loro dominio e possesso». ${ }^{82}$ L'arcivescovo Guidi chiede però che anche Roma faccia la sua parte, incaricando il nunzio apostolico di Vienna perché incontri l'imperatore e lo faccia desistere dal proposito di sostenere i greci «scismatici», dietro i quali — sospetta l'arcivescovo — si cela sicuramente il «ministro russo alla corte di Vienna». ${ }^{83}$

L'episodio livornese è spia di una strategia che la Russia di Caterina adotta nei confronti dei greci in diaspora in tutti i Paesi europei. Nel Regno di Sardegna sabaudo, proprio per favorire relazioni diplomatico-commerciali con la Russia si pensa di aprire i porti di terraferma e concedere le terre della spopolata Sardegna a mercanti e contadini greco-ortodossi. ${ }^{84}$ È l'ennesimo segnale del nesso che lega diplomazia, diaspora e colonizzazione interna, in un vincolo che, come nel caso di Minorca, fa di ogni colonia diasporica il terminale di una rete di relazioni, policentrica e trans-frontaliera.

del commercio e la difesa delle prerogative della Corona. Viene abolito il 2I settembre I765 con l'avvento di Pietro Leopoldo.

8I. ASPF, SC, Italo-Greci, b. 4 (I74I-I760), c. 35I.

82. Ibidem.

83. Ibidem.

84. Archivio di Stato di Torino (ASTO), Corte, Materie politiche per rapporto all'estero, Corti Straniere, Russia, mazzo I. Alla trattativa si accenna in SALICE, Colonizzazione sabauda e diaspora greca, pp. 187-89. 


\section{La dissoluzione}

La Russia sfrutta le potenzialità strategiche di Minorca fintanto che l'isola resta sotto il controllo britannico ed è abitata dalla colonia greca. La conquista spagnola del I78I mette fine a tutto questo. Gli spagnoli espellono i forestieri che hanno difeso la Gran Bretagna e sequestrano i loro beni. ${ }^{85}$ Come già successo nel 1756 , i greci si disperdono, questa volta definitivamente. Il 3 I agosto 1785 viene consegnata al capitano generale dell'isola la lista delle famiglie greche che ancora si trovano a Minorca. Tra i pochi greci rimasti ci sono il mercante Giorgio Ladico (che dice di abitarvi dal 1753) con sua moglie e sette figli (due maschi e cinque femmine) ${ }^{86}$ C'è poi Antonio Rayle, cittadino britannico, residente a Minorca da 35 anni, che ha due figli e fa commercio in Villa Carlos. ${ }^{87}$ Nicola Ciro è invece arrivato da Livorno da appena un mese per vendere cappotti e dichiara che vi si tratterrà per quattro o cinque mesi. Anastasio Riva, nativo di Cefalonia, vive a Minorca da sei anni circa, mentre Juan Georgio Calerico vi abita da circa trent'anni. Tutti i greci menzionati si professano cattolici e romani, anche se alcuni ammettono di praticare il culto orientale. Non sappiamo se simili dichiarazioni siano sincere o se gli interrogati mentano per non essere espulsi dall'isola tornata sotto un sovrano cattolico.

Chi non ha timore di dichiarare la propria fede ortodossa e l'obbedienza al patriarca di Costantinopoli sono gli esponenti della famiglia Alexiano ancora residenti a Minorca. ${ }^{88}$ Callista, a Mahón da trent’anni,

85. Sulle confische delle case abbandonate da inglesi, greci ed ebrei in San Filippo cfr. AHM, Reial Patrimoni (Rpa), 78.6.

86. AHM, UM, 390. S.n.

87. Si tratta di Georgetown, fondata dai britannici a Minorca nel I77I. Cfr. Joan-Enric Vilardell Santacana, La fundación de Georgetown, Menorca, I77r: Patrick Mackellar y el urbanismo militar británico, Col-legi Oficial d'Arquitectes de les Illes Balears, Palma de Mallorca, 2005.

88. AHM, UM, 390. S.n. 
è a capo di un gruppo familiare formato da sole donne, le sue sei nipoti. Donne che attendono l'arrivo di Panioto Alexiano, fratello di Callista che risiede da tempo a Livorno, come console britannico e comandante di marina russo. Sarà proprio Panioto a portarle finalmente via dall'isola.

Queste presenze sono gli ultimi segni di vita di una colonia mortalmente colpita dalla riconquista spagnola di Minorca. Anche gli Alexiano hanno subito perdite economiche molto pesanti. A nulla è valsa la petizione che nel 1782 un ormai anziano Nicolas Alexiano, capitano del porto e capo della colonia greca fino al termine della seconda dominazione britannica, ha presentato alle autorità spagnole, nella speranza di incassare i 650 dollari che gli sarebbero dovuti per il nolo delle sue proprietà. ${ }^{89}$ Dalle indagini condotte dall'assessore Segui su mandato di Louis de Crillon, è emerso che il minorchino Diego Ernandes aveva effettivamente consegnato circa 657 dollari di Nicolas Alexiano a don Bernardo Idalgo, procuratore fiscale dell'esercito. L'assessore ha inoltre dimostrato che ad Alexiano appartenevano anche il vino e il sale presenti nel magazzino del capitano Gabriele Tuduri e vari altri beni caricati su diversi vascelli.

Quella tracciata da Segui è comunque una mappa incompleta delle confische che hanno colpito la famiglia guida della colonia greca. E tuttavia, il I8 febbraio il duca di Crillon dichiara che il greco-britannico Nicolas Alexiano non ha nulla da pretendere nell'isola, se non gli effetti strettamente personali lasciati al castello di San Filippo in seguito alla capitolazione. ${ }^{\circ \circ} \mathrm{La}$ sentenza segna la rovina per Nicolas, che morirà poco tempo dopo, lasciando la moglie Anna Chiesa Matrones, minorchina e cattolica apostolica romana, con sei figli maschi, tutti battezzati nella chiesa «scismatica» dei greci. ${ }^{91}$ Finisce così un matrimo-

89. NA, CO, I74, I, c. 314.

90. Ibidem.

9I. Dopo la confisca del patrimonio e la morte del marito, Anna e i suoi figli trovano riparo nella casa del padre, don Joseph Chiesa, che li mantiene. Cfr. AHM, UM, 390. s.n. 
nio che ci rivela come anche in un quadro sociale tormentato come quello minorchino, così segnato dagli scontri tra locali e forestieri, tra cattolici e ortodossi, ci fosse spazio per l'integrazione e la mescolanza tra patrimoni, culture, lingue e religioni diverse.

Un'integrazione divenuta però impraticabile nella Minorca tornata in mani spagnole, nella quale non è più possibile professare liberamente e pubblicamente il culto ortodosso. La chiesa «scismatica» di San Nicola viene confiscata immediatamente. ${ }^{92}$ I greci fanno appena in tempo a salvare gli arredi sacri, gli oggetti e le icone appartenute al tempio, per poi affidarli, nel I782, alla chiesa della Santissima Trinità di Livorno. Una buona parte di quegli arredi era stata donata ai greco-minorchini dalla zarina Caterina II di Russia nel 1769-I770. ${ }^{93}$ Le relazioni globali che hanno accompagnato la nascita e l'intera vita della colonia mercantile e scismatica di Minorca marcano così anche l'attimo più drammatico della sua storia, quello nel quale si consuma la sua definitiva dissoluzione.

92. Sulle confische disposte dal procuratore fiscale patrimoniale delle case abbandonate da inglesi, greci ed ebrei nel castello di San Filippo si veda AHM, RPa 78.6 .

93. Francisco Protonotari, Nuova antologia, I866-78 (200I), p. 345. 